\title{
Calidad de vida en cuidadores de pacientes con Diabetes Mellitus e Hipertensión Arterial en Centro de Salud-Benito Juárez, Hidalgo
}

Quality of life in caregivers of patients with Diabetes Mellitus and Hypertension in "Health

Center -Benito Juárez", Hidalgo

\author{
Karina Chávez-Rubio ${ }^{a}$, Alma B. López-Escudero ${ }^{b}$, José Arias-Rico ${ }^{c}$, Reyna .C. Jiménez- \\ Sánchez ${ }^{d}$, Viridiana Ramírez-Gutiérrez ${ }^{e}$, Roberto L. Méndez Ledezma ${ }^{f}$, Osmar A. Jaramillo- \\ Morales $^{g}$, María L. Sánchez-Padilla ${ }^{g}$, Margarita Lazcano-Ortiz ${ }^{g}$
}

\begin{abstract}
:
Objective: To determine the factors that influence the quality of life of the primary caregiver in patients with chronic noncommunicable diseases: Diabetes Mellitus and Arterial Hypertension of the Health Center of the Benito Juárez locality in San Agustín Tlaxiaca, Hidalgo. Material and method: Design of the non-experimental study, of descriptive cross-sectional quantitative type. The sample was 12 people, Primary caregivers of patients with chronic noncommunicable diseases: Diabetes Mellitus and Arterial Hypertension in the town of Benito Juárez, San Agustín Tlaxiaca. An instrument was applied that information economic, social and psychological data of the primary caregiver, which is made up of 21 items. Results: it was observed that the quality of life of the primary caregiver of patients with chronic noncommunicable diseases: diabetes mellitus and arterial hypertension in the Benito Juárez health center, is affected by the different factors that are identified in relation to their quality of life, which are linked to the repercussions that the disease, the treatments of its patients bring with it, in the physical, psychological and socioeconomic order.
\end{abstract}

Keywords:

Caregiver, Diabetes Mellitus, Arterial Hypertension, Quality of life.

\begin{abstract}
Resumen:
Objetivo: Determinar los factores que influyen en la calidad de vida del cuidador primario en pacientes con enfermedades crónico no transmisibles: Diabetes Mellitus e Hipertensión Arterial del Centro de Salud de la localidad Benito Juárez en San Agustín Tlaxiaca, Hidalgo. Material y método: Diseño del estudio no experimental, de tipo cuantitativo descriptivo transversal. La muestra fue de 12 personas, Cuidadores primarios de pacientes con enfermedades crónico no transmisibles: Diabetes Mellitus e Hipertensión Arterial de la localidad de Benito Juárez, de San Agustín Tlaxiaca. Se aplicó un instrumento que valora datos económicos, sociales y psicológicos del cuidador primario, el cual está conformado por 21 ítems. Resultados: se observó que la calidad de vida del cuidador primario de pacientes con enfermedades crónicas no transmisibles: diabetes mellitus e hipertensión arterial en el centro de salud Benito Juárez, se ve afectada por los distintos factores que se identifican en relación con su calidad de vida, los cuales se ven vinculados con las repercusiones que la enfermedad, los tratamientos de sus pacientes traen consigo, en el orden físico, psicológico y socioeconómico.
\end{abstract}

Palabras Clave:

Cuidador, Diabetes Mellitus, Hipertensión Arterial, Calidad de vida.

\footnotetext{
Autor de Correspondencia, Universidad Autónoma del Estado de Hidalgo, Instituto de Ciencias de la Salud, ORCID: 0000-0001-5719-1854, Email:kary070895@ gmail.com ${ }^{b}$ Universidad Autónoma del Estado de Hidalgo, Instituto de Ciencias de la Salud, ORCID: 0000-0003-4578-6441, Email: belem_0924@ hotmail.com Universidad Autónoma del Estado de Hidalgo, Instituto de Ciencias de la Salud, ORCID: 0000-0003-0219-0410. Email: jose_arias@uaeh.edu.mx Universidad Autónoma del Estado de Hidalgo. Instituto de Ciencias de la Salud. ORCID: 0000-0001-9264-8514, Email: cristyji@ hotmail.com Universidad Autónoma del Estado de Hidalgo. Instituto de Ciencias de la Salud, Email: viri_gutierrez16@ hotmail.com

${ }_{\mathrm{f}}^{\mathrm{f}}$ Universidad Autónoma del Estado de Hidalgo. Instituto de Ciencias Básicas e Ingeniería, Email: robertomendez2197@ @otmail.com

${ }^{g}$ Profesores de la Universidad Autónoma del Estado de Hidalgo. Instituto de Ciencias de la Salud, Email: oajmorales@gmail.com.
} 


\section{Introducción}

La Organización Mundial de la Salud (OMS) define la calidad de vida como "la percepción que un individuo tiene de su lugar en la existencia en el contexto de la cultura y del sistema de valores en los que vive y en relación con sus expectativas, sus normas y sus inquietudes. Se trata de un concepto muy amplio que está influido de modo complejo por la salud física del sujeto, su estado psicológico, su nivel de independencia, sus relaciones sociales, así como su relación con los elementos esenciales de su entorno" (OMS, 2016). Es necesario entender la calidad de vida como un concepto multidimensional, con un gran componente subjetivo, que se refleja en una percepción general de satisfacción.

La calidad de vida del cuidador primario es de vital importancia ya que es el responsable de realizar las acciones de cuidado a los pacientes con enfermedades crónicas dependientes, de tal forma que al brindar el cuidado este sufre complicaciones, como son ansiedad, depresión, estrés y fatiga. Sauras Jorge expresa que "La calidad de vida de las personas que cuidan a los pacientes con enfermedades crónicas es un parámetro de suma importancia ya que tiene efectos en el bienestar tanto físico, psicológico, social y económico de las personas que los cuidan". (Sauras, 2009).

Los cuidadores primarios ocupan un lugar esencial en la atención de estos enfermos absorbiendo la carga principal de los cuidados que se le brindan al enfermo, teniendo como consecuencia el "síndrome del cuidador primario". Este se relaciona con la carga que, a nivel físico y emocional, padecen quienes se ocupan de este desgastante papel. Ser un cuidador no es un trabajo fácil, muchas veces la responsabilidad de cuidar a un paciente con dependencia grave requiere de tiempo completo, conocimiento y disposición, esto implica un desgaste físico, emocional, económico, entre otros, que puede modificar la calidad de vida del mismo (Facultad de Enfermería, 2016).

La diabetes es una enfermedad crónica que aparece cuando el páncreas no produce insulina suficiente 0 cuando el organismo no utiliza eficazmente la insulina que produce. La insulina es una hormona que regula el azúcar en la sangre, en 2014 el 8,5\% de los adultos (18 años o mayores) tenía diabetes. En 2015 fallecieron 1,6 millones de personas como consecuencia directa de la diabetes y los niveles altos de glucemia fueron la causa de otros 2,2 millones de muertes en 2012 (OMS, Organización Mundial de la Salud, 2016).

La tensión arterial es la fuerza que ejerce la sangre contra las paredes de los vasos (arterias) al ser bombeada por el corazón. Cuanto más alta es la tensión, más esfuerzo tiene que realizar el corazón para bombear (OMS, Organización Mundial de la Salud, 2016).

El objetivo de este trabajo fue determinar los factores que influyen en la calidad de vida del cuidador primario en pacientes con enfermedades crónicas no transmisibles: Diabetes Mellitus e Hipertensión Arterial del Centro de Salud de la localidad Benito Juárez en San Agustín Tlaxiaca, Hidalgo.

\section{Metodología}

Se utilizó como instrumento de valoración una encuesta que valora los datos económicos, sociales y psicológicos del cuidador primario, el instrumento cuenta con 21 ítems. La muestra fue de 12 personas. El tipo de investigación es de tipo cuantitativo de diseño no experimental, descriptivo ya que describe las características más específicas de fenómeno tal como se presenta en la realidad.

Este trabajo fue abalado por el comité de ética del área académica de enfermería del ICSa.

\section{Resultados}

De la población se obtuvo que el $49.9 \%$ están casados(as), $24.9 \%$ son solteros, $16.6 \%$ son divorciados y $8.3 \%$ en una situación de unión libre (tabla 1), también que, el $33.3 \%$ tienen un nivel de escolaridad de primaria, $41.6 \%$ de secundaria, $8.3 \%$ terminaron la educación media superior y el $16.6 \%$ el nivel superior (tabla 2 )

Al analizar los resultados observamos que las personas que respondieron a estas interrogantes refieren que su estado económico se ve afectado, ya que su ingreso económico es bajo y este no es suficiente para cubrir en su totalidad el tratamiento médico y las necesidades de su familiar al cual le brinda el cuidado, así mismo las propias del cuidador (tabla 3).

Tabla 1. Nivel de Estudios

\begin{tabular}{ccc}
\hline & No. & Porcentaje \\
\hline Primaria & 4 & $33.3 \%$ \\
\hline Secundaria & 5 & $41.6 \%$ \\
\hline Media Superior & 1 & $8.3 \%$ \\
\hline Nivel Superior & 2 & $16.6 \%$ \\
\hline $\begin{array}{l}\text { Fuente: Instrumento } \\
\text { cuidadores de pacientes con Diabetes Mellitus } \\
\text { e Hipertensión Arterial" }\end{array}$
\end{tabular}

Tabla 2. Estado Civil

\begin{tabular}{lcc}
\hline & No. & Porcentaje \\
\hline Soltero & 3 & $24.9 \%$ \\
\hline Unión Libre & 1 & $8.3 \%$ \\
\hline Casado & 6 & $49.9 \%$ \\
\hline Divorciado & 2 & $16.6 \%$ \\
\hline
\end{tabular}

Fuente: Instrumento "Calidad de vida en cuidadores de pacientes con Diabetes Mellitus e Hipertensión Arterial"

Al evaluar los resultados obtenidos observamos que el cuidador piensa que debido al tiempo que dedica a su familiar no tiene el suficiente para su vida personal y que ha disminuido su intimidad, de igual forma su vida social se ha visto afectada negativamente y en ocasiones se siente incómodo por distanciarse de sus amistades (tabla 4). 
Tabla 3. Datos Económicos (\%)

\begin{tabular}{|c|c|c|c|c|c|}
\hline & Nunca & Raramente & $\begin{array}{c}\text { Algunas } \\
\text { Veces }\end{array}$ & $\begin{array}{c}\text { Bastante a } \\
\text { menudo }\end{array}$ & $\begin{array}{l}\text { Varias } \\
\text { veces }\end{array}$ \\
\hline $\begin{array}{l}\text { ¿Siente que el dinero } \\
\text { no le alcanza para } \\
\text { todo lo que requiere su } \\
\text { familiar? }\end{array}$ & & 0 & 18.2 & 36.4 & 5.5 \\
\hline $\begin{array}{l}\text { ¿Piensa que no tiene } \\
\text { suficientes ingresos } \\
\text { económicos para los } \\
\text { gastos de cuidar a su } \\
\text { familiar, además de } \\
\text { otros gastos? }\end{array}$ & 0 & 9.1 & 0 & 72.7 & 8.2 \\
\hline
\end{tabular}

Tabla 4. Datos Sociales (\%)

\begin{tabular}{|c|c|c|c|c|c|}
\hline & Nunca & Raramente & $\begin{array}{c}\text { Algunas } \\
\text { Veces }\end{array}$ & $\begin{array}{c}\text { Bastante a } \\
\text { menudo }\end{array}$ & $\begin{array}{l}\text { Varias } \\
\text { veces }\end{array}$ \\
\hline $\begin{array}{l}\text { ¿Piensa que debido al tiempo que } \\
\text { dedica a su familiar no tiene } \\
\text { suficiente tiempo para su vida? }\end{array}$ & 0 & 9.1 & 18.2 & 36.4 & 36.4 \\
\hline $\begin{array}{l}\text { ¿Piensa que no tiene tanta } \\
\text { intimidad como le gustaría debido } \\
\text { a tener que cuidar de su familiar? }\end{array}$ & 0 & 0 & 36.4 & 54.5 & 9.1 \\
\hline $\begin{array}{l}\text { ¿piensa que su vida social se ha } \\
\text { visto afectada negativamente por } \\
\text { tener que cuidar a su familiar? }\end{array}$ & 0 & 0 & 36.4 & 63.6 & 0 \\
\hline $\begin{array}{l}\text { ¿se siente incómodo por } \\
\text { distanciarse de sus amistades } \\
\text { debido a tener que cuidar de su } \\
\text { familiar? }\end{array}$ & $\begin{array}{ll}r \\
s \\
4\end{array}$ & 0 & 27.3 & 54.5 & 18.2 \\
\hline
\end{tabular}

Tabla 5. Datos Psicológicos (\%)

\begin{tabular}{lccccc}
\hline \multicolumn{1}{l}{ Nunca } & Raramente & $\begin{array}{c}\text { Algunas } \\
\text { veces }\end{array}$ & $\begin{array}{c}\text { Bastante } \\
\text { a menudo }\end{array}$ & $\begin{array}{c}\text { Varias } \\
\text { veces }\end{array}$ \\
\hline $\begin{array}{l}\text { Globalmente, ¿Qué grado de } \\
\text { carga experimenta por el hecho } \\
\text { de cuidar a su familiar? }\end{array}$ & 0 & 1 & 36.4 & 4.5 & 0 \\
$\begin{array}{l}\text { ¿Piensa que su familiar } \\
\text { depende de usted? }\end{array}$ & 0 & 0 & 0 & 6.4 & 3.4 \\
$\begin{array}{l}\text { ¿Se siente tenso cuando está } \\
\text { cerca de su familiar? }\end{array}$ & 0 & 0 & 36.4 & 4.5 & .1 \\
$\begin{array}{l}\text { ¿Siente que ha perdido el } \\
\text { control de su vida desde que } \\
\text { comenzó la enfermedad de su } \\
\text { familiar }\end{array}$ & 0 & 0 & 54.5 & 8.2 & 27.3 \\
$\begin{array}{l}\text { ¿Desearía poder dejar el } \\
\text { cuidado de su familiar a otra } \\
\text { persona? }\end{array}$ & 18.2 & 36.4 & 5.5 & 0 \\
\hline
\end{tabular}

Fuente: Instrumento "Calidad de vida en cuidadores de pacientes con Diabetes Mellitus e Hipertensión Arterial"

Al observar los datos psicológicos obtenidos globalmente; el cuidador refiere que experimenta un alto nivel de carga por el hecho de brindar un cuidado, presentando emociones tensas, ya que piensa que su familiar depende en su totalidad del, esto trae como consecuencia sentimientos de perder el control y bastante a menudo preferiría designar el cuidado de su familiar a otra persona (tabla 5).

\section{Discusión}

En esta investigación encontramos que el $72 \%$ de los participantes pertenecen al género femenino una cifra que se acerca a la arrojada en la tesis "Sobrecarga del cuidador primario de pacientes con enfermedades crónico degenerativas", en donde además encontramos que también existe una similitud en el estado civil ya que la cifra de esta investigación identificados como casados fue de $54.5 \%$, mientras que en el porcentaje de la tesis de referencia fue de $57 \%$. En otro punto de comparación encontramos que el $54.5 \%$ experimenta un grado de sobrecarga por el hecho de cuidar a su familiar a diferencia del escrito de referencia que fue de $52 \%$ (Pineda, 2015).

En otro escrito llamado "carga y dependencia de cuidadores primarios informales de pacientes con parálisis cerebral infantil severa" observamos que existen altos porcentajes de resultados negativos relacionados al estado psicológico de cuidador entre ellos encontramos: grado de impotencia, tristeza, angustia y más, como también una carga de estrés en los encuestados del $53.4 \%$ qué comparamos con el resultado de $36.4 \%$ de algunas veces a la pregunta de ¿qué grado de carga experimenta por el hecho de cuidar a su familiar?, en lo analizado por Ariza y Milena también observamos que las relaciones sociales y familiares se ven afectadas ya que maneja factores relacionados con nuestra investigación uno más de estos ejemplos en la afectación negativa del cuidador con otros miembros de la familia, aquí el $82 \%$ manifestó un cambio relacionado con los miembros de su núcleo, que al contraponer con nuestro resultado encontramos un resultado de $63.6 \%$ que respondieron bastante a menudo a la pregunta ¿piensa que su vida social se ha visto afectada negativamente por tener que cuidar a su familiar? (López, 2015).

\section{Conclusión}

El resultado general del estudio calidad de vida del cuidador primario de pacientes con enfermedades crónicas no transmisibles: diabetes mellitus e hipertensión arterial en el centro de salud Benito Juárez, se ve afectada por los distintos factores que se identifican en relación con su calidad de vida, los cuales se ven vinculados con las repercusiones que la enfermedad, los tratamientos de sus pacientes traen consigo, en el orden físico, psicológico y socioeconómico.

Dados los resultados es evidente que los cuidadores requieren atención inmediata para mejorar su calidad de vida, puesto que es preocupante que más de la mitad de los cuidadores sin distinción de sexo y edad se encontraron comprometidos respecto a su calidad de vida.

\section{Referencias}

1) Andrews, R., \& Whitney, A. (2006). Psiquiatría. Obtenido de Desarrollo historico del concepto de calidad de vida: https://www.unioviedo.es/psiquiatria/wpcontent/uploads/2017/03/1993_Bobes_Desarrollo.pdf

2) Dan, V. (2005). Revista Medica Electronica. Obtenido de Calidad de vida: evolucion historica,perspectivas y consideraciones.: https://www.revista-portalesmedicos.com/revista-medica/calidad-devida-evolucion-historica/

3) Electrónica, R. M. (2014). Revista Medica Electronica. Obtenido de Calidad de vida: evolucion historica,perspectivas y consideraciones.: https://www.revista-portalesmedicos.com/revista-medica/calidad-devida-evolucion-historica/

4) Española, A. d. (2014). Real Academia Española. Obtenido de Real Academia Española: http://dle.rae.es/?id=6nVpk8P

5) Española, R. A. (2016). Diccionario de la Lengua Eespañola. Obtenido de http://dle.rae.es/?id=BbmCLvC

6) Facultad de Enfermería, U. d. (2016). Aquichan. Obtenido de Aquichan:

http://aquichan.unisabana.edu.co/index.php/aquichan/article/view/87/ 179

7) Garduño Estrada, L. (2006). Calidad de vida y bienestra subjetivo en México.

Trillas. 
Publicación semestral, Educación y Salud Boletín Científico Instituto de Ciencias de la Salud Universidad Autónoma del Estado de

Hidalgo, Vol. 8, No. 15 (2019) 165-168

8) Gordon, M. (2000). Pasado, Presente y futuro de los diagnosticos de enfermería. Ref Works.

9) Guadalupe, L. A. (2006). Psicología de la salud y calidad de vida. Paraninfo. INSP. (2017). Secretraria de Salud. Obtenido de https://www.insp.mx/

10) Levi, L. (2008). RevistA Latinoamericana de Psicologia. Obtenido de Calidad de vida: una definicion integradora: http://www.redalyc.org/pdf/805/80535203.pdf

11) López, C. R. (15 de 04 de 2015). Red CDPD. Obtenido de Red CDPD: http://repositoriocdpd.net:8080/handle/123456789/904

12) Oblitas, L. A. (2006). Psicología de la salud y calidad de vida Paraninfo.

13) OMS. (2016). Organizacion Mundial de la Salud. Obtenido de Organizacion Mundial de la Salud: http://www.who.int/topics/es/

14) PND. (2013). Plan Nacional de Desarrollo. Obtenido de Plan Sectorial de Salud: http://www.conadic.salud.gob.mx/pdfs/sectorial_salud.pdf

15) República, G. d. (2013). Plan Nacional de Desarrollo. Obtenido de Plan Sectorial de Salud: http://www.conadic.salud.gob.mx/pdfs/sectorial_salud.pdf

16) Sauras, J. (2009). Secretaría de estado de educación y formación profesional. España: Truller.

17) Szalai, A. (2014). The meaning of corporative research on the quality of life. London: Comparative Studies. 